\title{
Different inhibition characteristics of intracellular transglutaminase activity by cystamine and cysteamine
}

\author{
Ju-Hong Jeon ${ }^{1 *}$, Hye-Jin Lee ${ }^{1 *}$, \\ Gi-Yong Jang ${ }^{1}$, Chai-Wan Kim ${ }^{1}$, \\ Dong-Myung Shin ${ }^{1}$, Sung-Yup Cho ${ }^{1}$, \\ Eui-Ju Yeo ${ }^{1 \#}$, Sang-Chul Park ${ }^{1}$ \\ and $\mathrm{In}-\mathrm{Gyu} \mathrm{Kim}^{1,2}$
}

\author{
${ }^{1}$ Department of Biochemistry and Molecular Biology/Aging \\ Apoptosis Research Center (AARC) \\ Seoul National University College of Medicine \\ Seoul 110-799, Korea \\ ${ }^{2}$ Corresponding author: Tel, 82-2-740-8248; \\ Fax, 82-2-744-4534; E-mail, igkim@plaza.snu.ac.kr \\ ${ }^{*}$ Theses authors contributed equally to this work. \\ "Present address: Department of Biochemistry, Gachon Medical \\ School.
}

Accepted 13 December 2004

Abbreviations: TGase, transglutaminase; BP, 5-(biotinamido) pentylamine; DTT, dithiothreitol; GSH, reduced glutathione; Cystamine, $\beta, \beta$ '-diaminodiethyl disulfide or 2,2'-dithiobisethanamine; Cysteamine, $\beta$-mercaptoethylamine or 2-aminoethanethiol; PBS, phosphate-buffered saline; PBST, phosphate-buffered saline containing $0.1 \%$ Tween 20; BSA, bovine serum albumin

\begin{abstract}
The treatment of cystamine, a transglutaminase (TGase) inhibitor, has beneficial effects in several diseases including CAG-expansion disorders and cataract. We compared the inhibition characteristics of cystamine with those of cysteamine, a reduced form of cystamine expected to be present inside cells. Cystamine is a more potent inhibitor for TGase than cysteamine with different kinetics pattern in a nonreducing condition. By contrast, under reducing conditions, the inhibitory effect of cystamine was comparable with that of cysteamine. However, cystamine inhibited intracellular TGase activity more strongly than cysteamine despite of cytoplasmic reducing environment, suggesting that cystamine itself inhibits in situ TGase activity by forming mixed disulfides.
\end{abstract}

Keywords: cystamine; cysteamine; inhibitor; polyamine; transglutaminase

\section{Introduction}

Transglutaminases (TGases) are a family of enzymes that catalyze the transamidation reaction between the $\gamma$-carboxyamide of a peptide-bound glutamine residue and the $\varepsilon$-amino group of a peptide-bound lysine residue or the primary amine group of a polyamine via forming a thioester acylenzyme intermediate at the active site cysteine (Lorand et al., 1984). Thus, TGase activity produces cross-linked proteins or amine conjugates.

At least 3 types of TGases (TGase 1, 2 and 3) are expressed in human brain (Cooper et al., 2002). Given the fact that the catalytic properties of TGases acting on selected glutamine residues generate protein aggregates, TGase activity has been implicated in a number of neurodegenerative disorders caused by an abnormal expansion of polyglutamine repeats, such as Dentatorubral-pallidoluysian atrophy, Spinobulbar muscular atrophy, Spinocerebellar ataxia and Huntington's disease (HD) (Cooper et al., 2002; Gentile et al., 2004). Recent reports showed that the treatment with cystamine, a TGase inhibitor, resulted in prolonged survival and decreased abnormal movements in a murine model of HD (Dedeoglu et al., 2002; Karpuj et al., 2002), suggesting a possible role for TGases in the pathogenesis of neurodegeneration. Thus, the development of TGase inhibitors based on the inhibition mechanism of cystamine could lead to novel pharmacological strategies for the treatment of neurodegenerative disorders.

Cystamine $\left(\beta, \beta^{\prime}\right.$-diaminodiethyl disulfide) is a thiolamine joined by a disulfide bridge between two cysteamine ( $\beta$-mercaptoethylamine), and has been used to inhibit TGase activity in both in vitro and in vivo assay systems. However, the inhibition mechanism of cystamine has not been clearly understood. Since cystamine may be converted to cysteamine in cytoplasmic reducing environment (Cooper et al., 2002; Gentile et al., 2004), it has been thought that cysteamine, not cystamine, is an intracellular form of TGase inhibitor. Nonetheless, it is not known as to whether the inhibitory effect of cysteamine on TGase activity is comparable with cystamine. In this study, we compared the inhibitory effects of cystamine with those of cysteamine on TGase activity and showed that cystamine is a more potent inhibitor of TGase than cysteamine in the intact cells. 


\section{Materials and Methods}

\section{Materials}

5-(biotinamido)pentylamine was obtained from Pierce and other chemicals were purchased from Sigma (St. Louis, MO). $\left[{ }^{14} \mathrm{C}\right]$ putrescine was obtained from PerkinElmer. All culture media were purchased from Invitrogen.

\section{Cell culture}

A549, MCF7, T47D, HepG2 and K562 cells were maintained in RPMI 1640 medium containing 10\% fetal bovine serum. Saos2, HEK293, SH-SY-5Y, HeLa, PC3M, HaCaT and DU145 cells were cultured in Dulbecco's modified Eagle's medium supplemented with $10 \%$ fetal bovine serum. HLE-B3 cells were grown in modified Eagle's medium containing 20\% fetal bovine serum. Penicillin $(100 \mathrm{U} / \mathrm{ml})$, streptomycin sulfate $(100 \mu \mathrm{g} / \mathrm{ml})$ and glutamine $(2 \mathrm{mM})$ were supplemented to all cell culture media. All cell lines were maintained in a humidified atmosphere with $5 \%$ $\mathrm{CO}_{2}$ at $37^{\circ} \mathrm{C}$.

\section{Production of human TGase 2 protein}

Recombinant human TGase 2 protein was produced using baculovirus expression system (Pharmingen, San Diego, CA) and a nickel-nitrilotriacetic acid column (Qiagen, Hilden, Germany) as previously described (Shin et al., 2004), monitoring TGase activity by $\left[{ }^{14} \mathrm{C}\right]$ putrescine incorporation assay (Jeon et al., 2002). Purified TGase 2 was dialyzed against a buffer (30 mM Tris- $\mathrm{Cl}, \mathrm{pH} 7.5,15 \mathrm{mM} \mathrm{NaCl}$ ) and stored at $-20^{\circ} \mathrm{C}$ in a buffer $(30 \mathrm{mM}$ Tris- $\mathrm{Cl}, \mathrm{pH} 7.5,15 \mathrm{mM}$ $\mathrm{NaCl}$ and $50 \%$ glycerol).

Microtiter plate assay for in vitro TGase activity Each well of a 96-well microtiter plate was coated with $\mathrm{N}, \mathrm{N}^{\prime}$-dimethylcasein at $10 \mu \mathrm{g} / \mathrm{ml}(100 \mu \mathrm{l} / \mathrm{well})$ in a buffer $(50 \mathrm{mM}$ Tris-Cl, $\mathrm{pH} 7.5,150 \mathrm{mM} \mathrm{NaCl}, 5$ $\mathrm{mM}$ EGTA, $5 \mathrm{mM}$ EDTA) for $1 \mathrm{~h}$ at room temperature. The wells were overcoated with $5 \%$ bovine serum albumin (BSA) in phosphate-buffered saline (PBS) for $1 \mathrm{~h}$ at room temperature. The wells were then washed three times with PBS containing $0.1 \%$ Tween 20 (PBST). TGase 2 (10 ng of purified protein) was added to each well which contains 5-(biotinamido)pentylamine $(\mathrm{BP} ; 0-20 \mu \mathrm{M})$ in the $100 \mu \mathrm{l}$ of a buffer $(50 \mathrm{mM}$

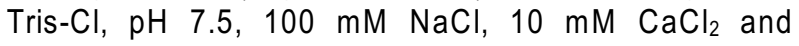
$0.1 \%$ Triton $\mathrm{X}-100)$. In experiments under reducing conditions, either dithiothreitol or glutathione at $0-20$ $\mathrm{mM}$, respectively, was included in buffer R. In experiments with TGase inhibitors, five amine compounds (cystamine, cysteamine, putrescine, spermidine and spermine) at $0-5 \mathrm{mM}$ were included in the reaction mixture. The plates were further incubated for $1 \mathrm{~h}$ at $37^{\circ} \mathrm{C}$, followed by washing three times with PBST. The incorporation of BP into N,N'-dimethylcasein was measured with horseradish peroxidase-conjugated streptavidin in $5 \%$ BSA $(1.25 \mathrm{mg} / \mathrm{ml}, 100 \mu \mathrm{l} /$ well $)$ for $45 \mathrm{~min}$ at $37^{\circ} \mathrm{C}$. After washing the cells with PBST five times, the wells of plates were incubated with O-phenylenediamine dihydrochloride $(0.4 \mathrm{mg} / \mathrm{ml}, 50$ $\mu \mathrm{l} /$ well) in citrate-phosphate solution $(0.1 \mathrm{M}$ citric acid, $0.2 \mathrm{M} \mathrm{Na}_{2} \mathrm{HPO}_{4}$ ) for 5-15 $\mathrm{min}$ at room temperature before the addition of $1 \mathrm{M} \mathrm{H}_{2} \mathrm{SO}_{4}(100 \mu \mathrm{l} /$ well $)$ solution. The color development was quantitated by measuring the absorbance at $490 \mathrm{~nm}$ on microplate spectrophotometer (Molecular Devices).

\section{in situ TGase assay}

in situ TGase activity was measured as previously described (Jeon et al., 2003a; b). Briefly, the cells (2 $\times 10^{6}$ ) were labeled with $\mathrm{BP}$ at $1 \mathrm{mM}$ or amine compounds (0 to $1.0 \mathrm{mM}$ ) in serum-free medium for $1 \mathrm{~h}$ prior to harvesting. After washing twice with PBS, the cells were suspended in PBS containing protease inhibitors and sonicated (2 s pulse/2 s pause $\times 5$, $20 \%$ amplitude). The homogenate was centrifuged for $10 \mathrm{~min}$ at $20,000 \mathrm{~g}$ at $4^{\circ} \mathrm{C}$. The cell extract $(0.2$ $\mathrm{mg} / \mathrm{ml}, 50 \mu /$ well) was coated in the wells of a 96-well microtiter plate for $12 \mathrm{~h}$ at $4^{\circ} \mathrm{C}$. The wells were then overcoated with $5 \%$ BSA in PBS for $1 \mathrm{~h}$ at room temperature. After washing three times with PBST, $\mathrm{BP}$ incorporated into the cellular proteins was assessed as performed in microtiter plate assays.

\section{Statistical analysis}

The mean values were compared using analysis of variance (one-way ANOVA) plus Student's $t$-test. Statistical significance was set at a $P$-value $<0.05$. GraphPad Softwares were used for statistical analysis and curve fitting.

\section{Results}

Inhibition of in vitro TGase activity by cystamine or cysteamine

To compare the difference of inhibition characteristics between cystamine and cysteamine in a reducing condition, we first examined the effects of reducing agents on in vitro TGase activity. Dithiothreitol (DTT) or reduced-glutathione (GSH) increases TGase activity at higher concentration (Figure $1 \mathrm{~A})$. The maximum increase of the TGase activity was 3.8-fold with DTT at a concentration of $10 \mathrm{mM}$, whereas the TGase activity was 2.7-fold increase with $\mathrm{GSH}$ at $5 \mathrm{mM}$. Interestingly, in contrast to DTT, the TGase activity with $\mathrm{GSH}$ higher than $5 \mathrm{mM}$ was remarkably diminished. 
A
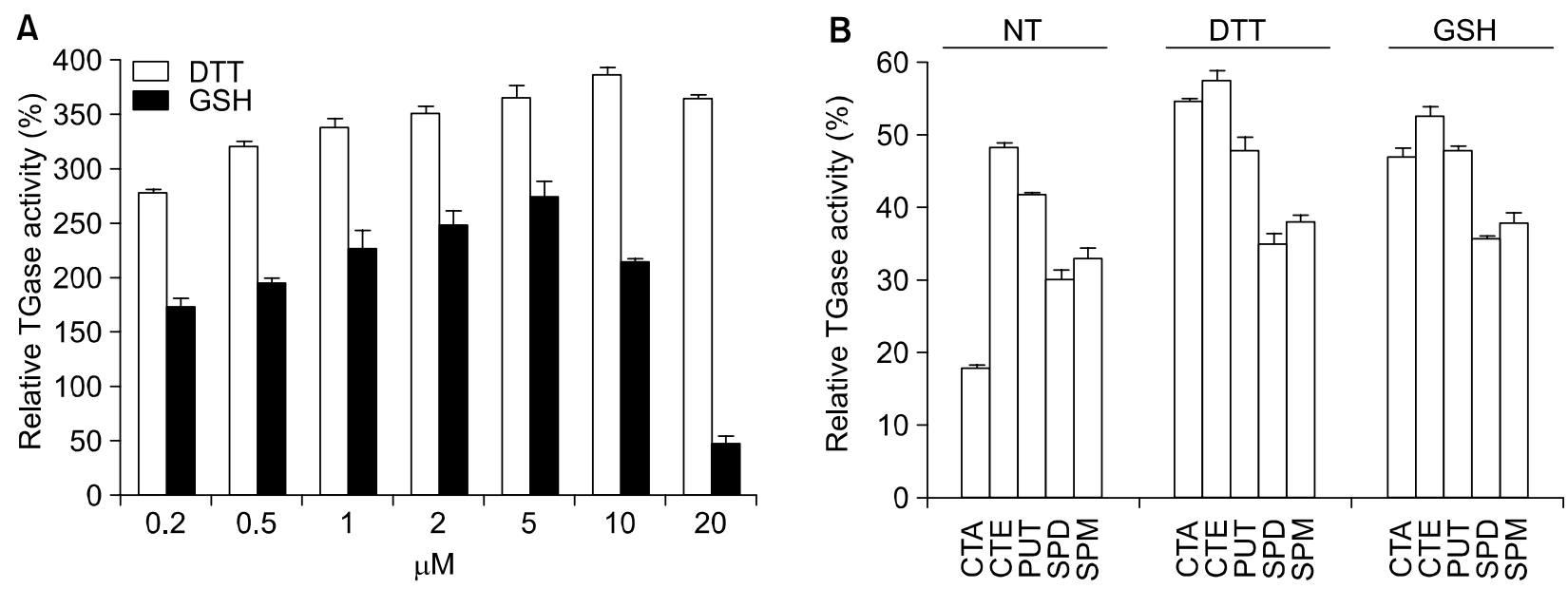

Figure 1. Inhibitory effects of cystamine and cysteamine on in vitro TGase activity. Recombinant TGase 2 (10 $\mathrm{ng}$ ) was incubated with BP (5 $\mu \mathrm{M})$ in the absence or presence of either DTT or GSH at various concentrations. The incorporation of BP to N,N'-dimethylcasein was measured with horseradish peroxidase-conjugated streptavidin and quantitated in terms of the absorbance at $490 \mathrm{~nm}$. Relative TGase activity is calculated based on the negative control, without DTT and GSH, as 100\%. (A) Effects of DTT or GSH on in vitro TGase activity. (B) Inhibition of in vitro TGase activity by amine compounds. Each amine compound $(200 \mu \mathrm{M}$; except cystamine at $100 \mu \mathrm{M}$ in the reaction with reducing conditions) was added to the reaction mixture. NT denotes the experimental condition without DTT and GSH. The figures represent the mean values \pm SEM. ( $n=3$ independent experiments performed with the samples in triplicate). CTA, cystamine; CTE, cysteamine; PUT, putrescine; SPD, spermidine; SPM, spermine.

Table 1. Inhibitory effects of cystamine or cysteamine on in vitro TGase activity in the presence of DTT or GSH.

\begin{tabular}{lccccc} 
& \multicolumn{4}{c}{$I_{50}(\mu \mathrm{M})$} \\
\cline { 2 - 5 } & NThibitors & DTT & $(\mathrm{DTT} / \mathrm{NT})$ & GSH & $(\mathrm{GSH} / \mathrm{NT})$ \\
\hline Cystamine & $22 \pm 5$ & $212 \pm 24$ & $(9.6)$ & $124 \pm 19$ & $(5.6)$ \\
Cysteamine & $178 \pm 17$ & $232 \pm 31$ & $(1.3)$ & $218 \pm 27$ & $(1.2)$
\end{tabular}

Recombinant TGase $2(10 \mathrm{ng})$ reacted with BP $(5 \mu \mathrm{M})$ in the absence (NT) or in the presence of either DTT or GSH at $2 \mathrm{mM}$, respectively. Cystamine or cysteamine at varying concentrations $(0-5,000 \mu \mathrm{M})$ was added to the reaction mixture. The table represents the mean values \pm SEM $(n=3) . \quad I_{50}$ denotes the inhibitory concentration resulting in a $50 \%$ inhibition of TGase activity.

We next compared the effects of cystamine and cysteamine on in vitro TGase activity in the presence or absence of reducing agents (Table 1). Cystamine and cysteamine inhibited in vitro TGase activity with $I_{50}$ values of 22 and $178 \mu \mathrm{M}$, respectively. In the presence of reducing agents, the inhibitory capacity of cystamine for TGase activity decreased by 5.6 -fold (GSH) or 9.6-fold (DTT). On the other hand, the inhibition by cysteamine decreased only slightly (1.2to 1.3-fold). These results indicate that cystamine is a more potent inhibitor for in vitro TGase activity than cysteamine in a non-reducing condition.

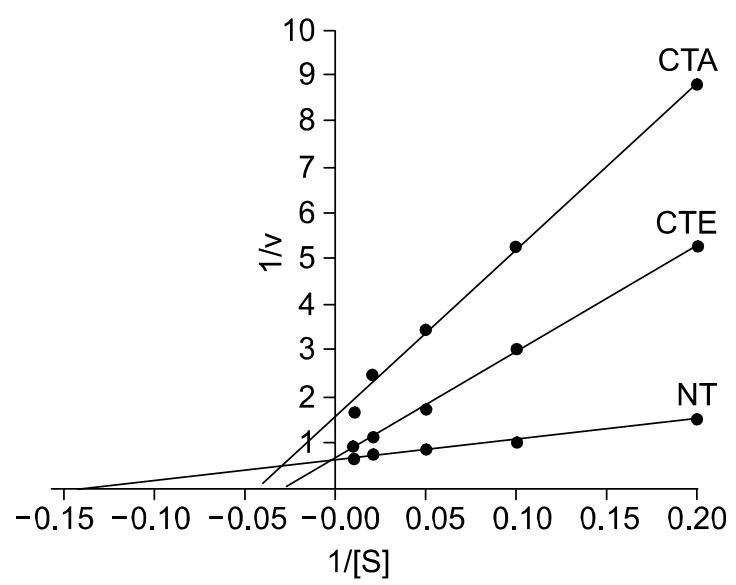

Figure 2. Kinetic analysis of TGase inhibition by cystamine or cysteamine under a non-reducing condition. Recombinant TGase 2 was incubated with varying concentrations of $\mathrm{BP}$ in the absence and presence of $500 \mu \mathrm{M}$ cystamine or cysteamine, respectively. The BP incorporated into $\mathrm{N}, \mathrm{N}$ '-dimethylcasein was measured with horseradish peroxidase-conjugated streptavidin by absorbance at $490 \mathrm{~nm}$. Representative data from a typical experiment are presented $(n=3$ independent experiments performed with the samples in duplicate).

\section{Comparison of inhibitory effects of cystamine with that of polyamines}

To determine whether the presence of disulfide bond of cystamine is associated with inhibitory capacity of cystamine, we compared the effects of naturally occurring polyamines including putrescine, spermidine and spermine, which have two primary amine groups 
without disulfide bond, on TGase with those of cystamine and cysteamine. Among five compounds examined under non-reducing condition, cystamine is the most, and cysteamine the least effective inhibitor of TGase activity with following order: cystamine > spermidine $\approx$ spermine $>$ putrescine $>$ cysteamine (Figure 1B). Putrescine, spermidine, spermine and cysteamine exhibited a similar inhibitory activity for TGase regardless of the presence of reducing agents. By contrast, only cystamine decreased its inhibitory capacity significantly under the reducing conditions.
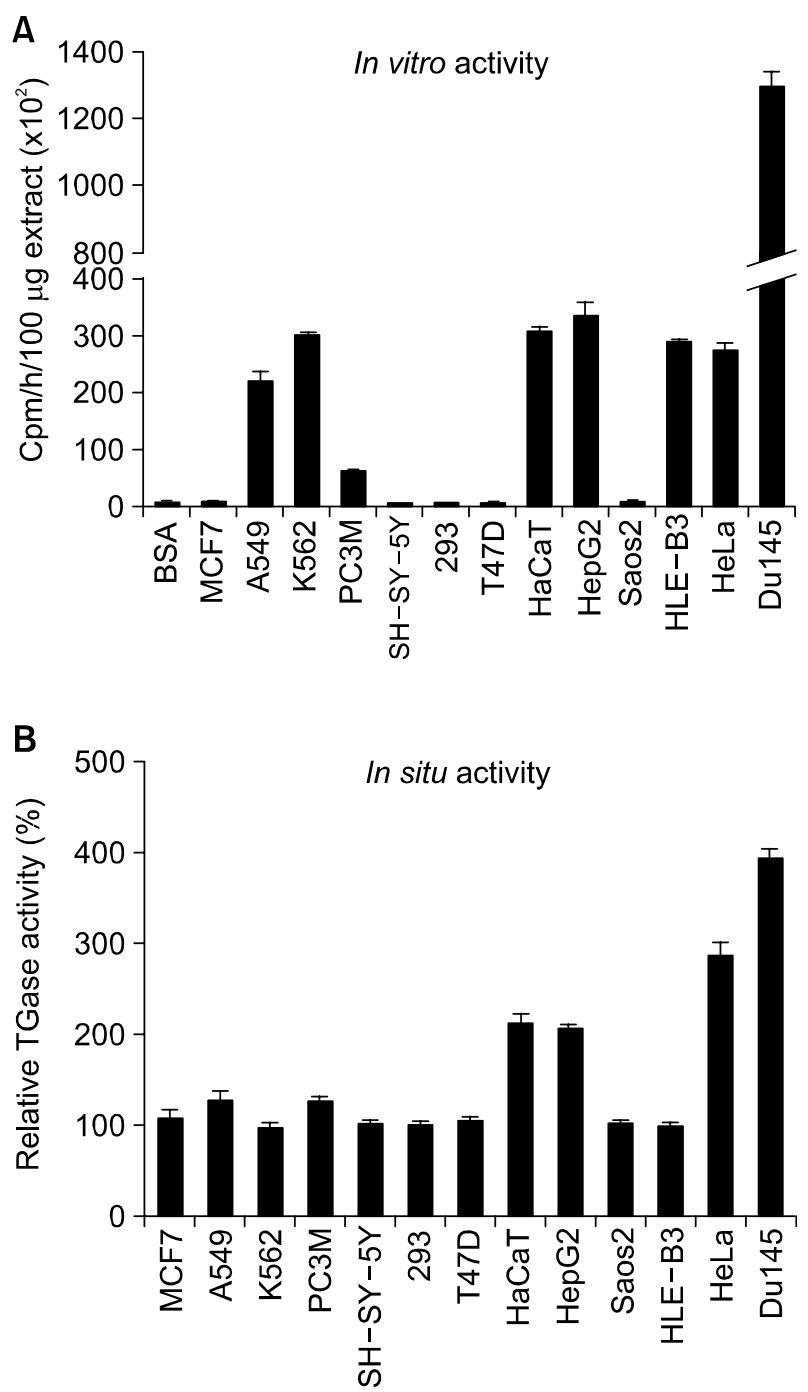

Figure 3. in vitro and in situ TGase activity of cultured cell lines. (A) in vitro TGase activity for each cell line was expressed in (cpm/h) per $100 \mu \mathrm{g}$ extract. BSA was adopted as a negative control for samples. The figures represent the mean values \pm SEM of three determinations. (B) The in situ TGase 2 activity of each cell line is expressed relative to that of control cells cultured in the absence of BP $(100 \%)$. The figures represent the mean values \pm SD of four determinations.
A Lineweaver-Burk plot showed that $\mathrm{Km}, \mathrm{Vmax}$ and slope were all affected by cystamine. The graph pattern seemed to be intermediate between those for competitive and non-competitive inhibitions. However, competitive inhibition pattern was noted only with cysteamine (Figure 2). By contrast, in the presence of reducing agents, both cystamine and cysteamine showed competitive inhibition kinetics (Cooper et al., 2002). Taken together, these results indicate that a disulfide bond of cystamine may react with the active site cysteine of TGase, which is required for the efficient inhibition of in vitro TGase activity.
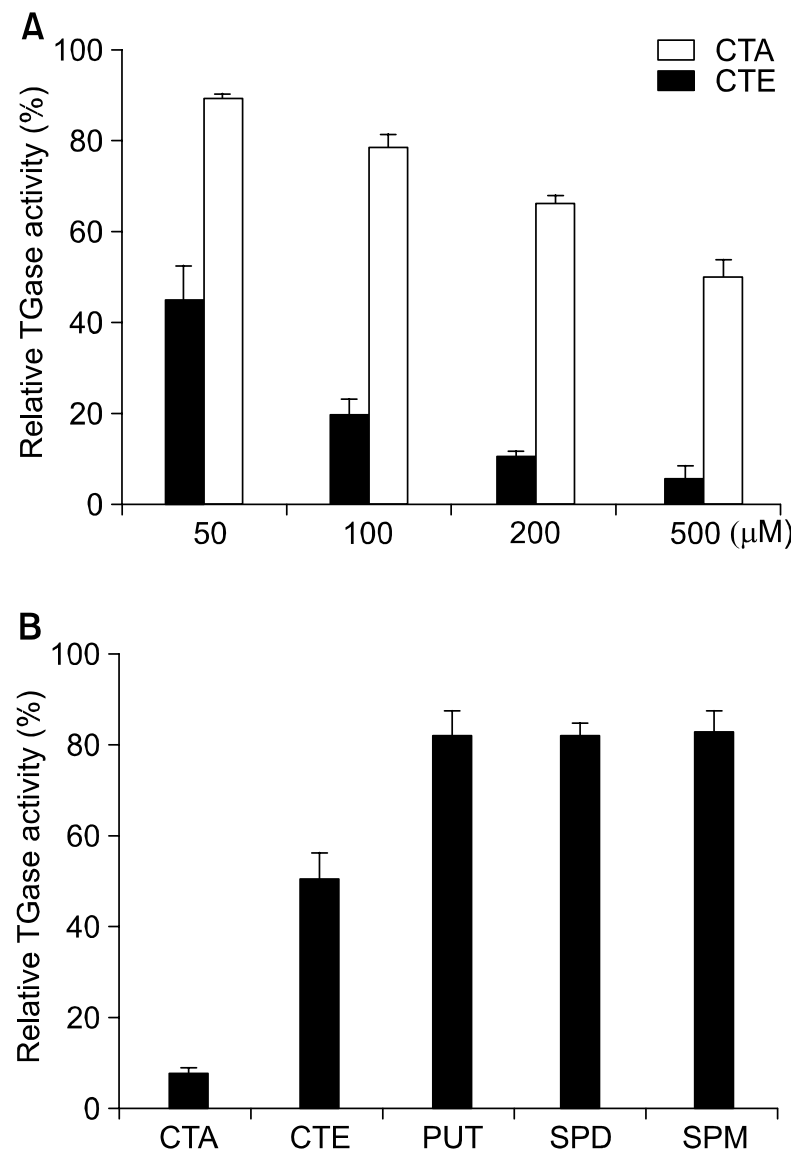

Figure 4. Inhibition of in situ TGase activity of DU145 cells by cystamine and cysteamine. (A) The inhibition of cellular in situ TGase activity with a various amount of cystamine or cysteamine. DU145 cells were labeled with BP in the presence of cystamine or cysteamine, respectively. (B) The inhibition of cellular in situ TGase activity with cystamine, cysteamine, putrescine, spermidine or spermine at $500 \mu \mathrm{M}$, respectively. TGase activity is expressed relative to that of the cells cultured without cystamine or cysteamine $(100 \%)$. The figures represent the mean values \pm SD $(n=3-4$ independent experiments performed with the samples in triplicate). The mean values were compared using analysis of variance (one-way ANOVA) plus Student's t-test. 


\section{Inhibition of in situ TGase activity by cystamine or cysteamine}

Cystamine may be converted to cysteamine in the cytoplasmic reducing environment (Cooper et al., 2002; Gentile et al., 2004). Thus, we questioned whether the inhibitory effect of cysteamine on in situ TGase activity is different from that of cystamine in cultured cell system. To address this question, a number of cell lines were screened in terms of in vitro and in situ TGase activities to select a suitable cell line. While A549, K562, PC3M, HepG2, HaCaT, HLEB3, HeLa and DU145 cells showed in vitro TGase activities (Figure 3A), HepG2, HaCaT, HeLa and DU145 cells exhibited remarkable intrinsic in situ TGase activities (Figure 3B). Among the cell lines examined, DU145 cells exhibited strong positive TGase activities both in vitro and in situ, and was selected for investigating in situ TGase activities.

Cystamine and cysteamine showed a concentration-dependent inhibition of in situ TGase activity of DU145 cells. However, it is apparent that cystamine is a more effective inhibitor than cysteamine (Figure $4 \mathrm{~A}$ ). When the inhibitory effects of five amine compounds on the in situ TGase activity were compared with each other, cystamine was found to be the most effective inhibitor (Figure 4B). These results indicate that in situ TGase activity may be inhibited by cystamine per se in cell culture experiments, and that cystamine may be not completely converted to cysteamine in the intracellular environment.

\section{Discussion}

Cystamine has been used as a TGase inhibitor in experiments in vitro as well as in vivo. It is proposed that cystamine inhibits TGase activity by forming mixed disulfide bonds that may block the formation of a thioester acylenzyme intermediate or by competing with transamidation reaction between the $\varepsilon$-amino group of a peptide-bound lysine residues and the $\gamma$-glutamyl residue of proteins bound to TGase (Lorand et al., 1979; Cooper et al., 2002). We found that the inhibition potency of cystamine is significantly decreased in the presence of reducing agents such as DTT or GSH, indicating that the disulfide bond of cystamine may be an important structural component in inhibiting TGase activity. Indeed, these data suggest that the formation of mixed disulfide bonds at active site cysteine residue of TGase mainly contribute to the inhibitory mechanism of cystamine. However, our kinetic studies suggest that the primary amine group of cystamine may also contribute to the competitive inhibition. These findings may be important for developing pharmacologic agents that can inhibit TGase activity in vivo.
The cytoplasmic environment is considered to be under a reducing condition (Cooper et al., 2002; Gentile et al., 2004). However, our results demonstrated that cystamine inhibited in situ TGase activity far more effectively than cysteamine. In addition, the current data indicate that the inhibitory capacity of cystamine was less affected by GSH than that by DTT, suggesting that cellular GSH redox regulation is not capable of effectively converting cystamine to cysteamine. However, since pharamacokinetic profiles, such as uptake rate and half-life, of thiolamines and polyamines were not examined in this study, we did not discriminate whether the differential effects between cystamine and cysteamine are caused by reducing capacity or pharmacokinetic parameters. The development of convenient assay system for detecting intracellular thiolamines is required to clarify these points.

In eukaryotic cells, three naturally occurring polyamines, putrescine, spermidine and spermine, are derived from L-arginine and L-methionine via a series of enzymatic reactions (Wallace et al., 2003). These polyamines are positively charged aliphatic cations. Since most polyamines in cells are sequestered by the association with negatively charged molecules, such as nucleic acids or phospholipids (Igarashi and Kashiwagi, 2000), only a small portion of free polyamines would be available as amine donors for TGase. Thus, this hypothesis may explain the poor capacity of natural polyamines for the inhibition of TGase activity in situ. Cysteamine is a small compound that contains one amine group and sulfhydryl group, and therefore, less prone to interact with other negatively charged molecules within cells. Thus, the availability of free amine group in the molecules may be a critical factor to determine its inhibitory capacity.

Despite positive therapeutic effects of cystamine in a murine model of HD (Dedeoglu et al., 2002; Karpuj et al., 2002), the mechanism by which cystamine treatment is beneficial to the mice is not understood. In addition to TGase inhibition, cystamine is able to replenish glutathione and to inhibit caspase activity (Lesort et al., 2003). Moreover, in contrast to cysteamine, it is known that cystamine does not cross the blood brain barrier (Cooper et al., 2002). Thus, it is probable that cystamine may act in symptomatic improvement of HD model mice by mechanism other than TGase inhibition, and that the metabolic effects of cysteamine could contribute to the therapeutic benefits in a murine model of HD.

Cysteamine is a metabolite of pantetheine or coenzyme $A$ and serves as a precursor for hypotaurine and taurine that is a neurotransmitter, exerting neuroprotective actions in neural tissue from ischemia, oxidative stress and the presence of free radicals (Vecsei et al., 1990; Saransaari et al., 2000). In 
addition, since cross-linking reaction between proteins is competitively inhibited by cysteamine (Lorand et al., 1984; Cooper et al., 2002), this inhibition leads to the accumulation of amine-conjugated proteins within the cells. Thus, the functional alteration of target proteins by thiolamine incorporation needs further characterization.

In summary, the inhibitory effects of cystamine and cysteamine on TGase activity were analyzed. The inhibitory capacity of cystamine in vitro was largely affected by the extent of the reduced form of the molecule. Nonetheless, cystamine inhibits in situ TGase activity decidedly stronger than cysteamine. These results may provide some insights for future investigation, regarding cystamine or its derivatives, to develop a treatment for TGase-related disorders.

\section{Acknowledgment}

We thank Dr. Y. D. Kim for critical comments on the manuscript. This work was supported by Korea Science and Engineering Foundation (KOSEF) through the Center for Aging and Apoptosis Research at Seoul National University (R11-2002-001-04003-0) and Seoul National University Hospital Research Fund (to E.J.Y;05-1996-008). J.H.J., G.Y.J, and S.Y.C. were supported by graduate program of BK21 project from Ministry of Education and Human Resources Development.

\section{References}

Cooper AJL, Jeitner TM, Gentile V, Blass JP. Cross linking of polyglutamine domains catalyzed by tissue transglutaminase is greatly favored with pathological-length repeats: does transglutaminase activity play a role in $(C A G)(n) / Q(n)$ expansion diseases? Neurochem Int 2002;40:53-67

Dedeoglu A, Kubilus JK, Jeitner TM, Matson SM, Bogdanov M, Kowall NW, Matson WR, Cooper AJL, Ratan RR, Beal MF, Hersch SF, Ferrante RJ. Therapeutic effects of cystamine in a murine model of Huntington's disease. J Neurosci 2002;22:8942-50

Gentile V, Cooper AJL. Transglutaminases - possible drug targets in human diseases. Curr Drug Targets CNS Neurol
Disord 2004;3:99-104

Igarashi K, Kashiwagi K. Polyamines: mysterious modulators of cellular functions. Biochem Biophys Res Commun 2000; 271:559-64

Jeon JH, Cho SY, Kim CW, Shin DM, Kweon JC, Choi KH, Park SC, Kim IG, GTP is required to stabilize and display transamidation activity of transglutaminase 2 . Biochem Biophys Res Commun 2002;294:818-22

Jeon JH, Kim CW, Shin DM, Kim K, Cho SY, Kwon JC, Choi KH, Kang HS, Kim IG. Differential incorporation of biotinylated polyamines by transglutaminase 2. FEBS Lett 2003a;534:180-4

Jeon JH, Choi KH, Cho SY, Kim CW, Shin DM, Kwon JC, Song KY, Park SC, Kim IG. Transglutaminase 2 inhibits Rb binding of human papillomavirus E7 by incorporating polyamine. EMBO J 2003b;22:5273-82

Karpuj MV, Becher MW, Springer JE, Chabas E, Youssef $S$, Pedotti R, Mitchell D, Steinman L. Prolonged survival and decreased abnormal movements in transgenic model of Huntington disease, with administration of the transglutaminase inhibitor cystamine. Nat Med 2002;8:143-9

Lesort M, Lee M, Tucholski J, Johnson GVW. Cystamine inhibits caspase activity. Implications for the treatment of polyglutamine disorders. J Biol Chem 2003;278:3825-30

Lorand L, Parameswaran KN, Stenberg P, Tong YS, Velasco PT, Jonsson NA, Mikiver L, Moses P. Specificity of guinea pig liver transglutaminase for amine substrates. Biochemistry 1979;18:1756-65

Lorand L, Conrad SM. Transglutaminases. Mol Cell Biochem 1984;58:9-35

Saransaari P, Oja SS. Taurine and neural cell damage. Amino Acids 2000;19:509-26

Shin DM, Jeon JH, Kim CW, Cho SY, Kwon JC, Lee HJ, Choi KH, Park SC, Kim IG. Cell type-specific activation of intracellular transglutaminase 2 by oxidative stress or ultraviolet irradiation: implications of transglutaminase 2 in agerelated cataractogenesis. J Biol Chem 2004;279:15032-9

Vecsei L, Widerlov E. Preclinical and clinical studies with cysteamine and pantethine related to the central nervous system. Prog Neuropsychopharmacol Biol Psychiatry 1990; 14:835-62

Wallace HM, Fraser AV, Hughes A. A perspective of polyamine metabolism. Biochem J 2003;376:1-14 